\title{
A systematic search for near-infrared counterparts of nearby ultraluminous X-ray sources (II)
}

\author{
K. M. López, ${ }^{1,2 \star}$ M. Heida ${ }^{3 \star}$ P. G. Jonker, ${ }^{1,2 \star}$ M. A. P. Torres, ${ }^{1,2}$ T. P. Roberts, ${ }^{4}$ \\ D. J. Walton, ${ }^{3}$ D.-S. Moon ${ }^{5}$ and F. A. Harrison ${ }^{3}$ \\ ${ }^{1}$ SRON Netherlands Institute for Space Research, NL-3584 CA Utrecht, the Netherlands \\ ${ }^{2}$ Department of Astrophysics/IMAPP, Radboud University, PO Box 9010, NL-6500 GL Nijmegen, the Netherlands \\ ${ }^{3}$ Space Radiation Laboratory, California Institute of Technology, Pasadena, CA 91125, USA \\ ${ }^{4}$ Department of Physics, Centre for Extragalactic Astronomy, University of Durham, South Road, Durham DH1 3LE, UK \\ ${ }^{5}$ Department of Astronomy and Astrophysics, University of Toronto, Toronto, ON M5S 3H4, Canada
}

Accepted 2017 April 3. Received 2017 April 3; in original form 2017 February 1

\begin{abstract}
We present the results of our continued systematic search for near-infrared (NIR) candidate counterparts to ultraluminous X-ray sources (ULXs) within $10 \mathrm{Mpc}$. We observed 42 ULXs in 24 nearby galaxies and detected NIR candidate counterparts to 15 ULXs. Fourteen of these ULXs appear to have a single candidate counterpart in our images and the remaining ULX has two candidate counterparts. Seven ULXs have candidate counterparts with absolute magnitudes in the range between -9.26 and $-11.18 \mathrm{mag}$, consistent with them being red supergiants (RSGs). The other eight ULXs have candidate counterparts with absolute magnitudes too bright to be a single stellar source. Some of these NIR sources show extended morphology or colours expected for active galactic nuclei (AGNs), strongly suggesting that they are likely stellar clusters or background galaxies. The RSG candidate counterparts form a valuable sample for follow-up spectroscopic observations to confirm their nature, with the ultimate goal of directly measuring the mass of the compact accretor that powers the ULX using binary Doppler shifts.
\end{abstract}

Key words: stars: black holes -infrared: stars.

\section{INTRODUCTION}

Ultraluminous X-ray sources (ULXs) are defined as point-like, offnuclear sources with an X-ray luminosity that exceeds $10^{39} \mathrm{erg} \mathrm{s}^{-1}$ (Feng \& Soria 2011), the Eddington luminosity of a $10 \mathrm{M}_{\odot}$ black hole (Colbert \& Miller 2006). These high luminosities have been explained in different ways. In the first scenario, a ULX could be a stellar mass black hole emitting anisotropically (King et al. 2001), or alternatively, at super-Eddington luminosity (Begelman 2002; Moon, Eikenberry \& Wasserman 2003; Gladstone, Roberts \& Done 2009). Examples of these are the neutron star ULXs (Bachetti et al. 2014; Israel et al. 2017a,b). Another possibility is that the black hole is more massive than the typical $10 \mathrm{M}_{\odot}$ stellar remnants seen in our own Galaxy, resulting in a higher Eddington luminosity. A different hypothesis is that some ULXs could be recoiling supermassive black holes (SMBHs) whereas some could instead be remnant black holes from a smaller galaxy that underwent a merger with a larger galaxy (Jonker et al. 2010). The latter scenarios assume that ULXs host massive $\left(10^{2}-10^{5} \mathrm{M}_{\odot}\right)$ black holes. We call these intermediate mass

\footnotetext{
^E-mail: k.m.lopez@ sron.nl (KML); mheida@ caltech.edu (MH); p.jonker @ sron.nl (PGJ)
}

black holes (IMBHs). The recent detection of gravitational waves from a binary black hole merger, where the estimated mass of the final black hole is $62 \mathrm{M}_{\odot}$ (Abbott et al. 2016), proves the existence of more massive black holes than stellar mass black holes previously observed (Orosz et al. 2007; Casares \& Jonker 2014). IMBHs could be the building blocks of SMBHs (Ebisuzaki et al. 2001) and if they exist, they could help explain the puzzling observation that even at $z>6$, SMBHs with masses $>10^{9} \mathrm{M}_{\odot}$ exist (Fan et al. 2000; Wu et al. 2015; Bañados et al. 2016). In some sources, the IMBH interpretation of ULX is supported by luminosities $\gtrsim 10^{41} \mathrm{erg} \mathrm{s}^{-1}$, as such are difficult to achieve with current models of highly superEddington accretion (Sutton et al. 2012).

However, there exists no definitive evidence for an IMBH yet. The most reliable way to determine the true nature of ULXs is to determine the mass of their accretors via a dynamical mass measurement. To date, the most reliable mass constraint is that of the neutron star ULX M82-X2 (Bachetti et al. 2014), estimated through the detection of pulsations in this source and the inference that the upper limit to the mass of a neutron star is $3 \mathrm{M}_{\odot}$ (Kalogera \& Baym 1996).

Several studies have focused on detecting optical counterparts to ULXs (e.g. Gutiérrez \& López-Corredoira 2006; Ptak et al. 2006; Roberts et al. 2011; Gladstone et al. 2013; Fabrika et al. 2015) and 
Table 1. Galaxies observed in the $H$ band with the LIRIS instrument on the WHT.

\begin{tabular}{|c|c|c|c|c|c|c|c|c|}
\hline Galaxy & $\begin{array}{c}\text { Date } \\
\text { observed }\end{array}$ & $\begin{array}{c}\text { Exposure } \\
\text { time }^{a} \\
(\mathrm{sec})\end{array}$ & $\begin{array}{c}\text { WCS } \\
\text { uncertainty } \\
\quad \text { (mas) }\end{array}$ & $\begin{array}{l}\text { Zero-point } \\
\text { magnitude } \\
\quad(\mathrm{mag})\end{array}$ & $\begin{array}{l}\text { Limiting } \\
\text { magnitude }^{d} \\
\quad(\mathrm{mag})\end{array}$ & $\begin{array}{c}\text { Average } \\
\text { seeing } \\
(\operatorname{arcsec})\end{array}$ & $\begin{array}{l}\text { Distance } \\
(\mathrm{Mpc})\end{array}$ & $\begin{array}{c}\text { Distance } \\
\text { Ref. }\end{array}$ \\
\hline NGC 4190 & 2015 April 5 & 3920 & $253 \pm 20$ & $23.03 \pm 0.08$ & $19.06 \pm 0.01$ & 1.3 & $2.83 \pm 0.28$ & A \\
\hline NGC 4559 & 2015 April 5 & 3060 & $203 \pm 20$ & $23.06 \pm 0.10$ & $18.73 \pm 0.22$ & 1.2 & $7.31 \pm 1.46$ & A \\
\hline NGC 5194 & 2015 April 5 & 1720 & $164 \pm 20$ & $23.09 \pm 0.09$ & $18.82 \pm 0.13$ & 1.1 & $9.05 \pm 0.24$ & $\mathrm{~B}$ \\
\hline NGC 4490 & 2015 April 8 & 2580 & $120 \pm 15$ & $22.36 \pm 0.13$ & $18.48 \pm 0.62$ & 0.8 & $7.80 \pm 0.62$ & $\mathrm{C}$ \\
\hline NGC 4485 & 2015 April 8 & 900 & $9.95 \pm 15$ & $22.61 \pm 0.06$ & $18.62 \pm 0.28$ & 0.7 & $8.91 \pm 0.89$ & A \\
\hline NGC 4625 & 2015 April 8 & 4000 & $447 \pm 15$ & $23.22 \pm 0.05$ & $19.65 \pm 0.05$ & 1.0 & $8.20 \pm 0.66$ & $\mathrm{C}$ \\
\hline NGC 4736 & 2015 April 8 & 1400 & $225 \pm 15$ & $22.27 \pm 0.26$ & $18.31 \pm 0.04$ & 0.8 & $4.59 \pm 0.37$ & A \\
\hline NGC 5457 & 2015 April 8 & 1600 & $317 \pm 15$ & $23.20 \pm 0.08$ & $19.92 \pm 0.28$ & 0.8 & $6.95 \pm 0.42$ & $\mathrm{~A}$ \\
\hline NGC 891 & 2016 January 25 & 2400 & $109 \pm 20$ & $23.27 \pm 0.03$ & $19.11 \pm 0.51$ & 1.0 & $9.12 \pm 0.73$ & A \\
\hline NGC 891 & 2016 January 25 & 3720 & $154 \pm 20$ & $23.32 \pm 0.02$ & $20.06 \pm 0.36$ & 1.0 & $9.12 \pm 0.73$ & A \\
\hline NGC 2403 & 2016 January 25 & 4000 & $251 \pm 20$ & $23.07 \pm 0.03$ & $18.08 \pm 0.22$ & 1.1 & $3.18 \pm 0.19$ & A \\
\hline NGC 3486 & 2016 January 25 & 3900 & $26.7 \pm 15$ & $22.96 \pm 0.09$ & $19.03 \pm 0.27$ & 1.2 & $7.40 \pm 0.59$ & $\mathrm{C}$ \\
\hline NGC 1042 & 2016 January 26 & 3500 & $168 \pm 15$ & $23.27 \pm 0.03$ & $19.55 \pm 0.35$ & 0.9 & $4.21 \pm 0.30$ & $\mathrm{D}$ \\
\hline NGC 2500 & 2016 January 26 & 3680 & $172 \pm 15$ & $23.40 \pm 0.05$ & $20.16 \pm 0.06$ & 0.8 & $10.10 \pm 0.81$ & $\mathrm{C}$ \\
\hline NGC 2903 & 2016 January 26 & 4200 & $319 \pm 15$ & $23.11 \pm 0.19$ & $20.17 \pm 0.07$ & 0.7 & $9.46 \pm 1.89$ & A \\
\hline NGC 3990 & 2016 January 26 & 3000 & $157 \pm 20$ & $23.34 \pm 0.06$ & $19.11 \pm 0.19$ & 0.9 & $10.05 \pm 1.41$ & $\mathrm{~A}$ \\
\hline IC 342 & 2016 January 27 & 3060 & $105 \pm 20$ & $22.99 \pm 0.02$ & $19.31 \pm 0.41$ & 0.7 & $2.73 \pm 0.19$ & A \\
\hline NGC 855 & 2016 January 27 & 3660 & $9.3 \pm 20$ & $21.34 \pm 0.01$ & $18.37 \pm 0.12$ & 0.8 & $8.83 \pm 1.24$ & $\mathrm{~A}$ \\
\hline NGC 3031 & 2016 March 26 & 3960 & $317 \pm 15$ & $23.63 \pm 0.03$ & $18.94 \pm 0.04$ & 0.7 & $3.61 \pm 0.22$ & A \\
\hline NGC 4594 & 2016 March 26 & 1600 & $170 \pm 15$ & $23.49 \pm 0.03$ & $19.99 \pm 0.09$ & 0.9 & $11.27 \pm 1.35$ & $\mathrm{~A}$ \\
\hline NGC 4258 & 2016 March 26 & 3020 & $829 \pm 15$ & $23.49 \pm 0.03$ & $20.03 \pm 0.27$ & 0.8 & $7.31 \pm 0.37$ & A \\
\hline NGC 4258 & 2016 March 26 & 3500 & $263 \pm 15$ & $23.49 \pm 0.03$ & $20.55 \pm 0.05$ & 1.0 & $7.31 \pm 0.37$ & A \\
\hline NGC 4631 & 2016 March 27 & 2800 & $497 \pm 15$ & $22.90 \pm 0.01$ & $18.53 \pm 0.27$ & 0.9 & $7.35 \pm 0.74$ & A \\
\hline NGC 5128 & 2016 March 27 & 3500 & $80 \pm 20$ & $22.90 \pm 0.01$ & $17.79 \pm 0.11$ & 1.2 & $3.66 \pm 0.22$ & A \\
\hline NGC 4517 & 2016 March 27 & 3480 & $180 \pm 15$ & $23.42 \pm 0.06$ & $19.19 \pm 0.51$ & 0.8 & $8.58 \pm 0.77$ & A \\
\hline NGC 3521 & 2016 March 28 & 4000 & $107 \pm 20$ & $23.51 \pm 0.04$ & $20.17 \pm 0.18$ & 0.9 & $14.19 \pm 2.84$ & $\mathrm{~A}$ \\
\hline NGC 5194 & 2016 March 28 & 3600 & $147 \pm 20$ & $23.29 \pm 0.04$ & $18.09 \pm 0.01$ & 1.0 & $9.05 \pm 0.24$ & $\mathrm{~B}$ \\
\hline NGC 5457 & 2016 March 28 & 3500 & $38.8 \pm 15$ & $23.74 \pm 0.06$ & $20.35 \pm 0.10$ & 0.9 & $6.95 \pm 0.42$ & $\mathrm{~A}$ \\
\hline NGC 5457 & 2016 March 28 & 2920 & $225 \pm 15$ & $23.24 \pm 0.06$ & $20.47 \pm 0.17$ & 0.9 & $6.95 \pm 0.42$ & A \\
\hline
\end{tabular}

Notes. ${ }^{a}$ Effective exposure time, not necessarily equal to the net exposure time (i.e. images taken during twilight, cloudy intervals). ${ }^{b}$ Uncertainty with respect to the reference catalogue. The first value is a statistical uncertainty, given by the GAIA tool, while the second value is a systematic uncertainty, and corresponds to the astrometic accuracy of the reference catalog: 20 mas for UCAC4 and 15 mas for 2MASS. ${ }^{c}$ Zero-point magnitude derived from the data, with respect to the 2MASS catalogue. ${ }^{d}$ See the text in Section 3.2 for how the limiting magnitude is defined. References: A: Tully et al. (2013) B: Tikhonov, Lebedev \& Galazutdinova (2015) C: Tully (1988) D: Tully et al. (2009).

on radio counterparts (e.g. Pérez-Ramírez et al. 2011). Since the optical counterparts are often faint $(V>24 \mathrm{mag})$, radial velocity studies have made use of emission lines. However, these attempts have encountered difficulties, as the emission lines originate in the accretion disc and/or the surrounding nebulae, but not the companion star itself.

Others have focused on absorption lines from the donor stars on the blue part of the spectrum (e.g. Motch et al. 2014), as the observed colours are consistent with that of blue mass donors. In addition, several ULXs are located in or near young star clusters (e.g. Fabbiano, Zezas \& Murray 2001; Roberts et al. 2002; Gao et al. 2003; Poutanen et al. 2013), and thus, a blue early-type donor star might be expected certainly for those ULXs (e.g. Jonker et al. 2012).

The association with young star clusters implies that some of the donor stars can be red supergiants (RSGs; Copperwheat et al. 2005, 2007; Patruno \& Zampieri 2008; Heida et al. 2014), which are very bright in the near-infrared (NIR) band. Therefore, Heida et al. (2014, hereafter H14) performed the first systematic search for NIR counterparts to nearby $(D<10 \mathrm{Mpc})$ ULXs. Observing 62 ULXs, they discovered 17 candidate NIR counterparts, 11 of which had an absolute magnitude consistent with that of an RSG (see Table A1). During initial spectroscopic follow-up, they discovered RSG counterparts to ULX RX J004722.4-252051 (in
NGC 253, Heida et al. 2015), ULX J022721+333500 (in NGC 925) and ULX J120922+295559 (in NGC 4136, Heida et al. 2016).

In this paper, we present the results of our continued systematic search for candidate RSG counterparts to ULXs within $10 \mathrm{Mpc}$ from our Galaxy. We describe the sample in Section 2 and the NIR observations and data reduction/photometry in Section 3. The $\mathrm{X}$-ray astrometric correction is explained in Section 4 and our results are presented and discussed in detail in Section 5. We end with the conclusions of our work in Section 6.

\section{SAMPLE}

Our sample consists of $45^{1}$ ULXs located in 24 galaxies within $10 \mathrm{Mpc}$ from our own Galaxy (see Table 1), since this is the maximum distance at which it is possible to take NIR spectra of an RSG with existing telescopes (H14). This imaging campaign almost completes the ULX sample within $10 \mathrm{Mpc}$ taken from the catalogues of Liu \& Bregman (2005); Liu \& Mirabel (2005); Winter,

\footnotetext{
${ }^{1}$ We took 45 ULXs from the catalogues below, but we revise the number to 42 as in two cases two entries are likely from the same source (see subsection 4.3), and one source is likely not a ULX (see subsection 5.3.1).
} 
Mushotzky \& Reynolds (2006); Swartz et al. (2004, 2011); Liu (2011); Walton et al. (2011) and Earnshaw et al. (in preparation). Six ULXs were observed before by H14, but we observed them again under better sky conditions and in the $H$ band instead of the $K$ band in five of the six cases.

\section{NIR OBSERVATIONS}

$H$-band imaging of regions of galaxies containing ULXs was obtained with the Long-slit Intermediate Resolution Infrared Spectrograph (LIRIS) mounted on the William Herschel Telescope. LIRIS has a field of view of $4.27 \times 4.27$ arcmin and a pixel scale of 0.25 $\operatorname{arcsec}$ pixel $^{-1}$. The observations were performed using seven or eight repetitions of a 5-point dither pattern where five images ( $20 \mathrm{~s}$ exposure per image) were taken at each point. Of the 24 galaxies, eight were observed in 2015 April, nine in 2016 January and nine ${ }^{2}$ in 2016 March (see Table 1, where the average seeing is provided, as a measure of the image quality during the observations).

\subsection{Data reduction}

The data reduction was performed using the THELI pipeline (Schirmer 2013). With THELI we produced a master flat to flatfield correct the data and we generate a sky background model, which is subsequently subtracted from the individual data frames. In order to detect sources in the images and to obtain astrometric solutions, THELI uses SExTRACTOR (Bertin \& Arnouts 1996) and SCAMP (Bertin 2006), respectively. The astrometric solution is obtained by matching the detected positions to sources from the 2 Micron All Sky Survey (2MASS; Skrutskie et al. 2006) or Position and Proper Motion Extended-L (PPXML; Roeser, Demleitner \& Schilbach 2010). The global astrometric solution is subsequently used for the coaddition of all the images using SWARP (Bertin et al. 2002).

In order to obtain accurate astrometric positions, we improved the accuracy of the global astrometric solution of the co-added images using the STARLINK tool GAIA, fitting at least five star positions from the fourth US Naval Observatory CCD Astrograph Catalog (UCAC4, Zacharias et al. 2013) or 2MASS (if the field of view did not have five sources in UCAC4) to build a local astrometric solution around the position of the ULX. The rms errors of the fits are listed in Table 1, indicated as World Coordinate System (WCS) uncertainties, where the intrinsic error of the catalogue with respect to the International Celestial Reference System (ICRS) is also indicated: 15 mas (systematic) for 2MASS and 20 mas (systematic) for UCAC4. We were not able to improve the astrometry for one galaxy, NGC 4258 (observed on 2016 March 26), since there were not enough reference stars in the vicinity ( $1 \mathrm{arcmin})$ of the ULX. For this galaxy, we indicate the uncertainty for the global astrometric solution that THELI provides.

\subsection{Photometry}

We used SEXTRACTOR for the source detection and photometry in every NIR image, making sure that each detection was more than $3 \sigma$ above the local background. We performed aperture photometry to determine instrumental magnitudes. As aperture size, we use the average full width at half maximum (FWHM) of the light distribution of point-like objects in each image determined with the

\footnotetext{
${ }^{2}$ Two galaxies from 2015 April were re-observed in 2016 January.
}

STARLINK tool GAIA. The photometric zero-points of our NIR images were measured by using isolated 2MASS objects in the field of view. These are given in Table 1. Instrumental magnitudes for all detected sources were converted to apparent magnitudes. Finally, we determined the absolute magnitudes of any candidate counterparts to ULXs taking the distances given in Table 1 . The $(1 \sigma)$ uncertainties on these values are estimated taking into account the distance uncertainty, the uncertainty in the determination of the zero-point magnitude and the uncertainty in the instrumental magnitude given by SEXTRACTOR.

We estimated the limiting magnitude of each NIR image. To do this, we made a histogram of the magnitudes of the detected objects and the peak value of that histogram is a measure of the completeness limit, which we conservatively take as a measure of the limiting magnitude. We fit a linear model to the bright source end of the histogram of the magnitudes of each NIR image and compare the peak value with the value given by the linear fit to estimate the uncertainty on the limiting magnitude (see Table 1).

\section{X-RAY ASTROMETRIC LOCALIZATION}

For 14 ULXs out of the 45 accurate positions existed in the literature (e.g. Liu 2011, Swartz et al. 2011, Heida et al. 2014). In addition, we were able to determine accurate positions for 19 sources using archival Chandra/ACIS observations and for nine sources using archival XMM-Newton observations. The observation IDs and the exposure times are detailed in Table 2. We explain the detailed analysis for these 28 sources in Sections 4.1 and 4.2.

For the remaining three ULXs, observed only by ROSAT High Resolution Imager (HRI), we were not able to determine a position with higher accuracy than the original one from Liu \& Bregman (2005).

\subsection{Chandra observations}

In an attempt to reduce the uncertainty on the X-ray position of the ULXs, we queried the Chandra archive. For all sources with archival Chandra observations, we used the task ACIS_PROCESS_EVENTS in CIAO (Fruscione et al. 2006) to reprocess the event files with the latest calibration files (CALDB version 4.7.2) taking into account whether the observations were made in the 'Faint' or 'Very Faint' mode. We then produced images from data in the $0.3-7 \mathrm{keV}$ energy range, on which we run the WAVDETECT task (Dobrzycki et al. 1999) to establish accurate positions of all X-ray sources in the field of view of the Chandra ACIS CCDs.

In order to try to further improve the knowledge of the location of the ULXs, we investigated whether we could apply a boresight correction (e.g. Jonker et al. 2010). For this we search for X-ray sources detected with WAVDETECT with more than $20 \mathrm{X}$-ray counts and that lie within 3 arcmin of the optical axis of the satellite. When found, we investigated whether counterparts in the Sloan Digital Sky Survey (SDSS; Pier et al. 2003) or 2MASS catalogue exist. We considered the SDSS or 2MASS source a counterpart to the $\mathrm{X}$-ray source if the offset between the catalogue coordinates and the X-ray source position is less than 1 arcsec. We then apply shifts in RA and Dec. to the X-ray coordinates using the WCS_UPDATE tool. Using this boresight correction procedure, we were able to reduce the uncertainty on the location of 15 ULXs. Crowding of the optical or NIR fields precluded us to match the X-ray source to a unique optical/NIR source in six cases.

For four ULXs, only ROSAT coordinates are available in the literature (ULXs with IDs 11, 40, 41 and 45 in Table 3). For these 
Table 2. ULXs for which we reduced the uncertainty on the localization, through applying a boresight correction to the Chandra data, correcting the $X M M-N e w t o n$ position with the SAS software or improving the ROSAT position identifying the ULX in a Chandra image.

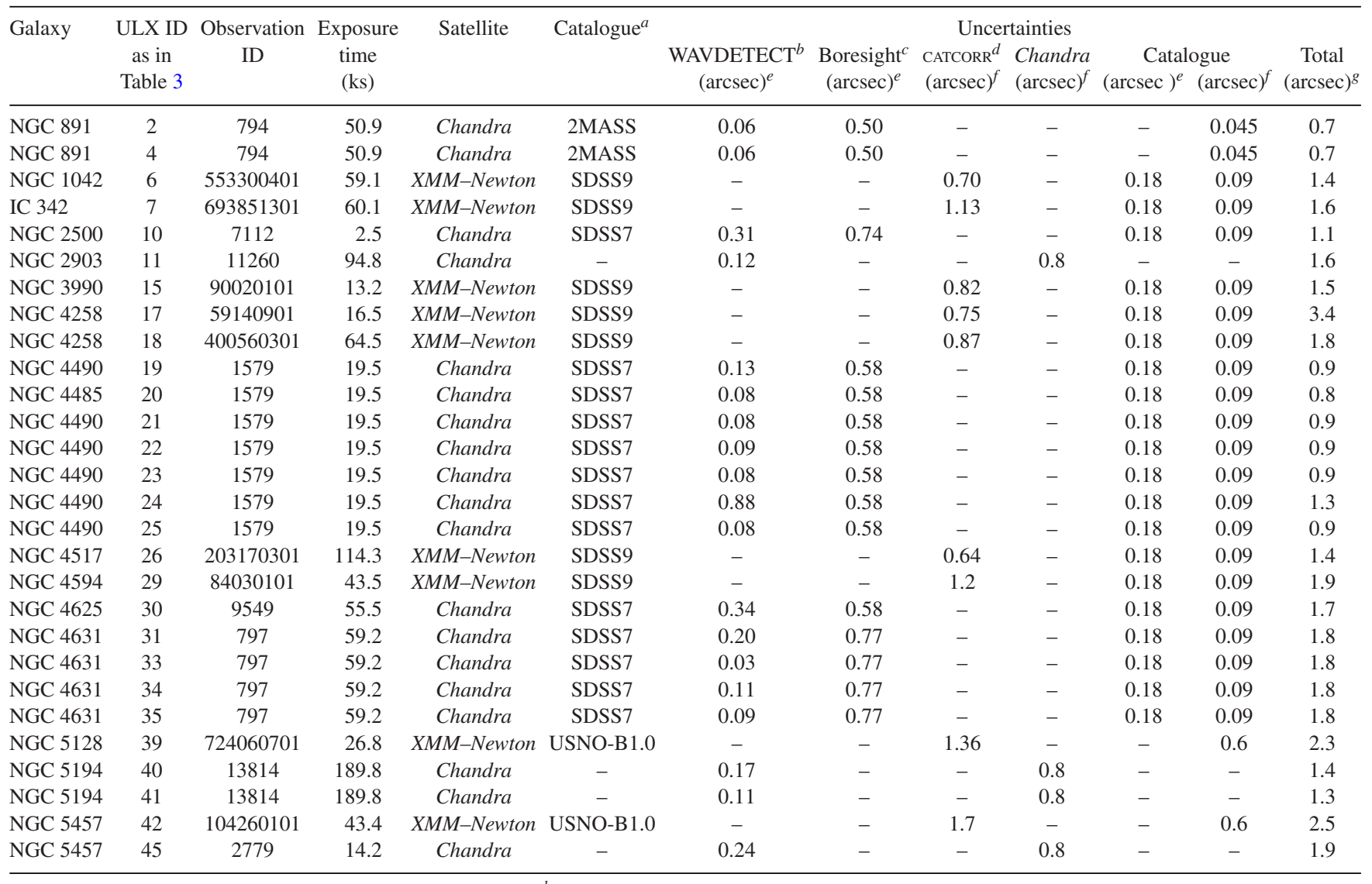

Notes. ${ }^{a}$ Catalogue used to perform the astrometric correction. ${ }^{b} 3 \sigma$ uncertainty in the source localization on the CCDs given by the WAVDETECT task. ${ }^{c} 3 \sigma$ uncertainty obtained after performing the boresight correction. ${ }^{d} 3 \sigma$ uncertainty after performing the astrometric correction for XMM-Newton data. ${ }^{e}$ Statistical uncertainty. ${ }^{f}$ Systematic uncertainty of the catalogue used (i.e. 0.8 arcsec for Chandra, Chandra X-ray Center). ${ }^{g}$ Calculated taking also into account the WCS uncertainty from the NIR image (see Table 1).

sources we provide the Chandra positions in Table 2. Even though we could not apply a boresight correction, those are more accurate than the ROSAT source positions from Liu \& Bregman (2005).

To evaluate the uncertainties in the final position of the ULX, we have to take into account: (a) the statistical uncertainty given by WAVDETECT in the source localization on the CCD and (b) the WCS uncertainty of the NIR image (see Table 1). In addition, for those sources where we applied a bore-sight correction, we must take into account (c) the statistical uncertainty between the corrected WAVDETECT position of the optical/infrared counterpart with its position in the SDSS/2MASS catalogues and (d) the instrinsic uncertainties of the SDSS/2MASS catalogues with respect to the ICRS: 3 mas (systematic) and 6 mas (statistical) for SDSS (Pier et al. 2003) and 15 mas for 2MASS. We added systematic uncertainties linearly and statistical uncertainties quadratically. The 99.7 percent $(3 \sigma)$ confidence radii for the ULXs final position are indicated in Table 3 .

\subsection{XMM-Newton observations}

Nine ULXs (ID 6, 7, 15, 17, 18, 26, 29, 39 and 42) were observed by $X M M-N e w t o n$ and therefore analysed with the Science Analysis System (SAS, XMM-Newton Data Analysis), using the CATCORR task. The SAS software updates the position of the sources by cross-matching the positions with three catalogues, SDSS, 2MASS and the US Naval Observatory (USNO-B1.0; Monet et al. 2003), to find optical or infrared counterparts and apply shifts or rotations to optimize the match.

The uncertainties of the final positions are derived with the systematic error given by CATCORR, which takes into account the uncertainty of the corrected position and the rotation of the field of view; the WCS uncertainty of the NIR image (from Table 1); and the intrinsic uncertainties of the SDSS/2MASS/USNO-B1.0 catalogues with respect to the ICRS: 0.03 and 0.06 arcsec for SDSS, 0.015 arcsec for 2MASS and 0.2 arcsec (systematic) for USNOB1.0 (Monet et al. 2003). The 99.7 per cent $(3 \sigma)$ confidence radii for the ULXs final position are given in Table 3.

\subsection{ROSAT observations}

There are three ULXs (ID 3, 5 and 32), which have been observed by the ROSAT mission but not by Chandra or XMM-Newton. Because of this, we could not improve the astrometry on these sources positions beyond that available in the literature. The $1 \sigma$ uncertainty on these positions is reported to be 3.62 arcsec by Liu \& Bregman (2005). The 99.7 per cent confidence radii for the these positions are provided in Table 3 and are calculated taking also into account the WCS uncertainty of the NIR image. 
Table 3. Complete sample of observed ULXs.

\begin{tabular}{|c|c|c|c|c|c|c|c|}
\hline ULX ID & Galaxy & $\begin{array}{l}\text { ULX name } \\
\left(\text { SIMBAD }^{a}\right)\end{array}$ & $\begin{array}{c}\text { RA } \\
\text { (hh:mm:ss) }\end{array}$ & $\begin{array}{c}\text { Dec. } \\
\text { (dd:mm:ss) }\end{array}$ & $\begin{array}{c}3 \sigma \text { position } \\
\text { uncertainty }^{b} \\
(\operatorname{arcsec})\end{array}$ & $\begin{array}{l}\text { Position } \\
\text { reference }{ }^{c}\end{array}$ & Telescope \\
\hline 2 & NGC 891 & [SST2011] J022231.26+421957.8 & $02: 22: 31.3$ & $42: 19: 57.4$ & 0.7 & This work & Chandra \\
\hline 3 & NGC 891 & [LB2005] NGC891 X1 & $02: 22: 31$ & $42: 20: 30^{e}$ & 11 & Liu \& Bregman (2005) & ROSAT \\
\hline 4 & NGC 891 & [PCV2006] ULX 2 & $02: 22: 31.4$ & $42: 20: 24.0^{e}$ & 0.7 & This work & Chandra \\
\hline 7 & IC 342 & [LB2005] PGC 13826 ULX2 & $03: 46: 45.4$ & $68: 09: 47.3$ & 1.6 & This work & $X M M-N e w t o n$ \\
\hline 8 & IC 342 & CXO J034657.4+680619 & $03: 46: 57.4$ & 68:06:19.1 & 0.9 & Evans et al. (2010) & Chandra \\
\hline 9 & NGC 2403 & RX J073655.7+653542 & $07: 36: 55.6$ & $65: 35: 40.8$ & 1.6 & Schlegel \& Pannuti (2003) & Chandra \\
\hline 10 & NGC $2500^{d}$ & [SST2011] J080148.10+504354.6 & 08:01:48.1 & $50: 43: 54.8$ & 1.1 & This work & Chandra \\
\hline 11 & NGC 2903 & [LB2005] NGC 2903 ULX1 & 09:32:01.9 & $21: 31: 11.1$ & 1.6 & This work & Chandra \\
\hline 12 & NGC $3031^{d}$ & [STS2009b] J095532.97+690033.4 & 09:55:32.9 & 69:00:33.6 & 1.8 & Liu (2011) & Chandra \\
\hline 18 & NGC 4258 & [LB2005] NGC 4258 X9 & $12: 19: 23.3$ & 47:09:40.4 & 1.8 & This work & $X M M-N e w t o n$ \\
\hline 19 & NGC 4490 & [SST2011] J123029.55+413927.6 & $12: 30: 29.5$ & $41: 39: 27.6$ & 0.9 & This work & Chandra \\
\hline 20 & NGC 4485 & RX J1230.5+4141 & $12: 30: 30.5$ & $41: 41: 42.1$ & 0.8 & This work & Chandra \\
\hline 21 & NGC 4490 & [SST2011] J123030.82+413911.5 & $12: 30: 30.7$ & $41: 39: 11.5$ & 0.9 & This work & Chandra \\
\hline 22 & NGC 4490 & [SST2011] J123032.27+413918.1 & $12: 30: 32.2$ & 41:39:18.1 & 0.9 & This work & Chandra \\
\hline 23 & NGC 4490 & [SST2011] J123036.32+413837.8 & $12: 30: 36.2$ & $41: 38: 37.9$ & 0.9 & This work & Chandra \\
\hline 24 & NGC 4490 & CXO J123038.4+413831 & $12: 30: 38.2$ & $41: 38: 31.1$ & 1.3 & This work & Chandra \\
\hline 25 & NGC 4490 & 2XMM J123043.1+413819 & $12: 30: 43.2$ & $41: 38: 18.5$ & 0.9 & This work & Chandra \\
\hline 26 & NGC 4517 & 3XMM J123242.7+000654 & $12: 32: 42.7$ & 00:06:54.9 & 1.4 & This work & $X M M-N e w t o n$ \\
\hline 27 & NGC 4559 & [SST2011] J123557.79+275807.4 & $12: 35: 57.8$ & 27:58:07.4 & 1.5 & Swartz et al. (2011) & Chandra \\
\hline 28 & NGC 4559 & RX J123558+27577 & $12: 35: 58.6$ & $27: 57: 41.9$ & 1.5 & Swartz et al. (2011) & Chandra \\
\hline 29 & NGC 4594 & [LB2005] NGC 4594 X5 & $12: 40: 22.6$ & $-11: 39: 25.2$ & 1.9 & This work & $X M M-N e w t o n$ \\
\hline 38 & NGC 4736 & CXO J125053.3+410714 & $12: 50: 53.3$ & 41:07:14.0 & 1.2 & Evans et al. (2010) & Chandra \\
\hline 39 & NGC 5128 & 2XMM J132538.3-430205 & $13: 25: 38.3$ & $-43: 02: 04.9$ & 2.3 & This work & $X M M-N e w t o n$ \\
\hline 40 & NGC 5194 & RX J132947+47096 & $13: 29: 47.5$ & $47: 09: 40.7$ & 1.4 & This work & Chandra \\
\hline 41 & NGC 5194 & [MEE95] R8 & $13: 29: 53.8$ & $47: 14: 35.8$ & 1.3 & This work & Chandra \\
\hline 42 & NGC 5457 & [LB2005] NGC 5457 X32 & $14: 01: 34.5$ & $+54: 20: 30.1$ & 2.5 & This work & $X M M-N e w t o n$ \\
\hline 43 & NGC 5457 & CXO J140303.9+542734 & 14:03:03.9 & $54: 27: 33.0$ & 0.8 & Evans et al. (2010) & Chandra \\
\hline 44 & NGC 5457 & CXO J140341.1+541903 & $14: 03: 41.2$ & 54:19:03.0 & 1.0 & Evans et al. (2010) & Chandra \\
\hline 45 & NGC 5457 & [LB2005] NGC 5457 X26 & 14:04:29.2 & $54: 23: 53.1$ & 1.9 & This work & Chandra \\
\hline
\end{tabular}

Notes. ${ }^{a}$ Set of Identifications, Measurements and Bibliography for Astronomical Data (SIMBAD; Wenger et al. 2000). ${ }^{b} 99.7$ per cent (3 $\sigma$ ) confidence radius around the position of the ULX within which we search for counterparts. ${ }^{c}$ Catalogue that provided the ULX position. ${ }^{d}$ Sources analysed by H14, that we re-observed in the $H$-band to provide deeper and better seeing images. ${ }^{e}$ ULXs that have the same position within a $2 \sigma$ confidence limit.

The position of two of the ULXs observed by ROSAT (NGC 4631 ID 32 and NGC 891 ID 3, from Liu \& Bregman 2005) coincides with the position of two unique ULXs observed by Chandra (NGC 4631 ID 33 and NGC 891 ID 4, from Swartz et al. 2011) to a 95 per cent $(2 \sigma)$ confidence level (see Fig. 1 ). For these two ULXs observed by Chandra, we performed a boresight correction (see Section 4.1), obtaining a 99.7 per cent confidence radii of 0.93 and 0.71 arcsec for NGC 4631 ID 33 and NGC 891 ID 4, respectively. Hence, it is likely that NGC 4631 ID 32 and ID 33 are the same source, as well as NGC 891 ID 3 and ID 4. For the detection of candidate counterparts for these two ULXs, we will use the Chandra localization.

\section{RESULTS AND DISCUSSION}

Of the 43 ULXs we observed, 15 have one NIR candidate counterpart and one has two candidate counterparts (see Fig. 2). Their apparent and absolute $H$-band magnitudes are detailed in Table 4. For the cases where no counterpart was detected, the limiting (absolute) magnitude of the NIR image is indicated. 


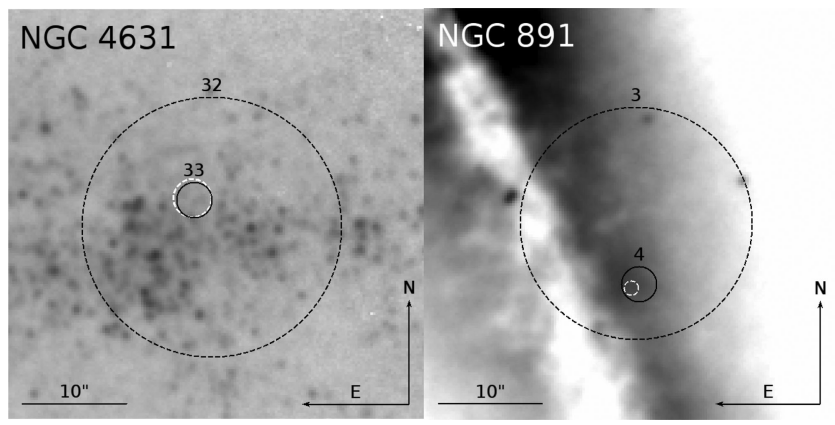

Figure 1. ROSAT (black dashed circle) and Chandra (black solid circle) positions of two ULXs on the NIR $H$-band image. The radii of the circles indicate the 99.7 per cent confidence level on the location of the ULX. The position of these ULXs coincides to a 95 per cent $(2 \sigma)$ confidence level; thus, we deem it likely that the ROSAT sources are the same as the Chandra sources and we remove the two ROSAT sources from our list. The white dashed circle indicates the position obtained after performing a boresight correction on the Chandra positions (see Section 4.1)

For the ULXs with candidate counterparts, we can investigate the nature of these by means of their absolute magnitude. Eight of the candidate counterparts have magnitudes ranging from -8.66 to $-11.18 \mathrm{mag}$, and within the uncertainties, these magnitudes are consistent with those of an RSG. The remaining nine candidate counterparts have absolute magnitudes in the range -11.44 to -14.85 mag, and are therefore too bright to be a single RSG. These sources are most likely background active galactic nuclei (AGN) or unresolved star clusters. However, we need to search for further evidence to determine their true nature. We want to stress that the only way to be certain of the classification of each candidate is to take spectra, but below we provide some clues on their possible nature.

For the 27 ULXs without a detected counterpart, the apparent limiting magnitudes range from 18.09-20.35 mag in the $H$ band. The limiting magnitudes brighter than 20 mag are mainly caused by crowded fields or high background from the host galaxies (i.e. NGC 4490, NGC 891, NGC 4736) and it will be difficult to improve on these using ground-based, natural seeing limited observations.

\subsection{Re-observed ULXs from H14}

We re-observed six ULXs previously studied in H14: NGC 855 ULX ID 1, NGC 2500 ULX ID 10, NGC 3031 ULX ID 12, NGC 3486 ULX ID 13, NGC 3521 ULX ID 14 and NGC 4258 ULX ID 17. We performed all our observations in the $H$ band, while $H 14$ took $K_{s}$-band images, with the exception of NGC 4258, which they also observed in the $H$ band. Our conclusions do not differ from H14 on ULX IDs 1, 10, 12 and 13. For the two other sources, we provide a more detailed assessment below.

\subsubsection{NGC 3521 ULX ID 14}

The 99.7 per cent confidence radius given by H14 for NGC 3521 is $2.1 \mathrm{arcsec}$, whereas our value is $1.6 \operatorname{arcsec}$ (see Fig. 2c). We detect a candidate counterpart (absolute $H$-band magnitude of $-10.93 \pm$ $0.93 \mathrm{mag}$ ), in contrast to the reported $K_{s}$ non-detection by $\mathrm{H} 14$. Our candidate has an absolute magnitude consistent with an Mtype supergiant. The limiting magnitude for our NIR image is $20.17 \pm 0.18 \mathrm{mag}$, and theirs is $19.25 \mathrm{mag}$; our average seeing is 0.9 arcsec and theirs is 0.7 arcsec. Due to the approximate $H-K=0$ colour or RSGs, our detection is consistent with their non-detection.

\subsubsection{NGC 4258 ULX ID 17}

Our 99.7 percent confidence radius around the ULX position is 3.4 arcsec, whereas the radius quoted in $\mathrm{H} 14$ is 2 arcsec. For this ULX, neither we nor H14 found enough astrometric standard stars (see subsection 3.1) local to the ULX, so we both used the global astrometric solution that THELI provides. The uncertainty on the position of the ULX is larger than that of $\mathrm{H} 14$, thus our 99.7 per cent confidence radius is larger. The average seeing in our NIR image is 0.8 arcsec, in contrast with the 0.7 arcsec seeing from $\mathrm{H} 14$. However, our limiting magnitude is almost 1 mag deeper than the one found by $\mathrm{H} 14$ and we find a candidate counterpart, whereas they indicated a non-detection. The detected candidate counterpart (see Fig. 2d) is located 2 arcsec from the centre of the ULX position, and has an absolute $H$-band magnitude of $-9.26 \pm 0.18$ mag, making it a potential RSG candidate. This $H$-band source lies outside the error radius from $\mathrm{H} 14$, but inside our 99.7 per cent confidence radius, hence we deem it a candidate counterpart. This ULX lies in the outskirts of the spiral arms of NGC 4258.

\subsection{NIR non-detections for ULXs with published searches for optical or radio counterparts}

Eight of the ULXs in our sample appear in searches for optical or radio candidate counterparts. Of these, we detect NIR candidate counterparts for three ULXs, which we discuss in detail in subsections 5.3 and 5.4. We do not detect any NIR candidate counterpart for the other five ULXs: IC 342 ULX ID 8, NGC 3031 ULX ID 12, NGC 4190 ULX ID 16, NGC 4490 ULX ID 21 and NGC 4736 ULX ID 36.

IC 342 ULX ID 8 was observed by Gladstone et al. (2013), where they detected one optical candidate counterpart. NGC 3031 ULX ID 12 was observed and analysed by Liu, Bregman \& Seitzer (2002) and Gladstone et al. (2013), and both reported an optical candidate counterpart. NGC 4190 ULX ID 16 was observed also by Gladstone et al. (2013), and they detected an optical candidate counterpart. Gutiérrez \& López-Corredoira (2006) observed NGC 4490 ULX ID 21 and detected a candidate counterpart H II region. In contrast, we do not detect any NIR candidate counterpart for these three ULXs. To investigate what our non-detections imply for the nature of the optical candidate counterparts, we need to be able to assess the probability that these optical and NIR sources are associated and for that one needs the coordinates of the optical candidate counterparts. Unfortunately, they are not given in the literature for these sources.

NGC 4736 ULX ID 36 was analysed by Gladstone et al. (2013), and was found not to have an optical candidate counterpart. We also find no NIR candidate counterpart.

\subsection{NIR red supergiant candidates}

\subsubsection{IC 342 ULX ID 7}

Just outside the 99.7 per cent confidence radius, there is a NIR candidate counterpart detected (see Fig. 2a), with an absolute magnitude of $-8.66 \pm 0.12$, consistent with the absolute magnitude of RSGs (Elias, Frogel \& Humphreys 1985; Drilling \& Landolt 2000). Visual inspection suggests that this source has several candidate counterparts inside the confidence radius; however, SExTRACTOR detected only a single object in this crowded area. 


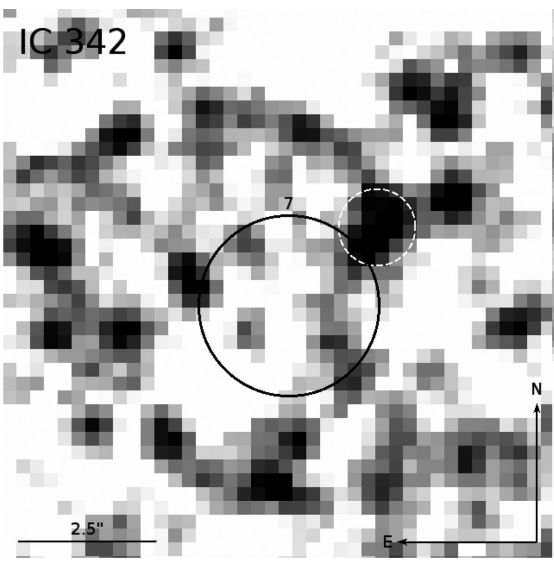

(a) IC 342 , ULX ID $7,3-\sigma=1.6$ "

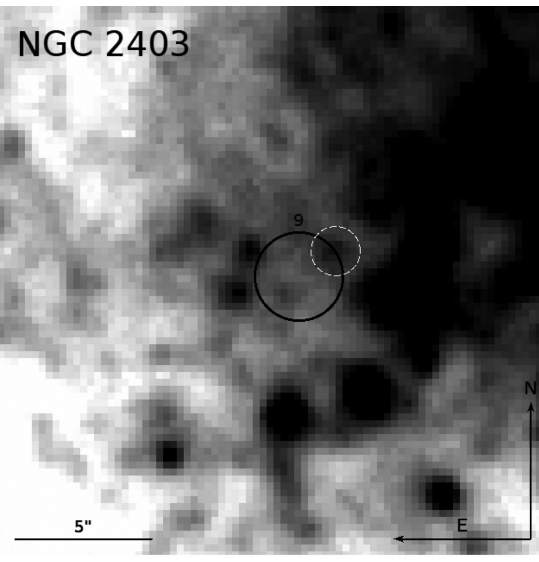

(b) NGC 2403, ULX ID 9, 3- $\sigma=1.6$ "

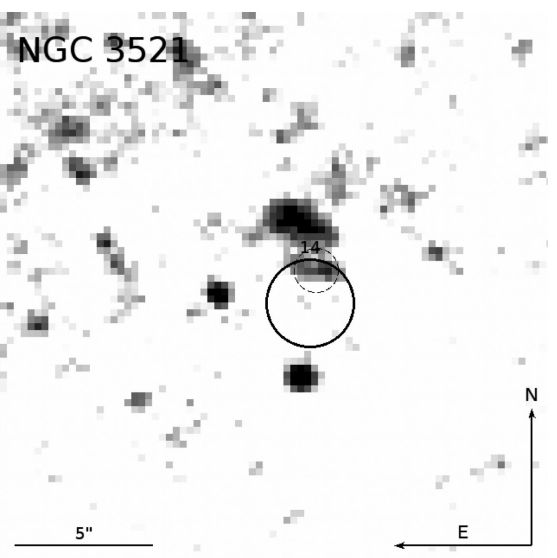

(c) NGC 3521, ULX ID 14, 3- $\sigma=1.6$ "

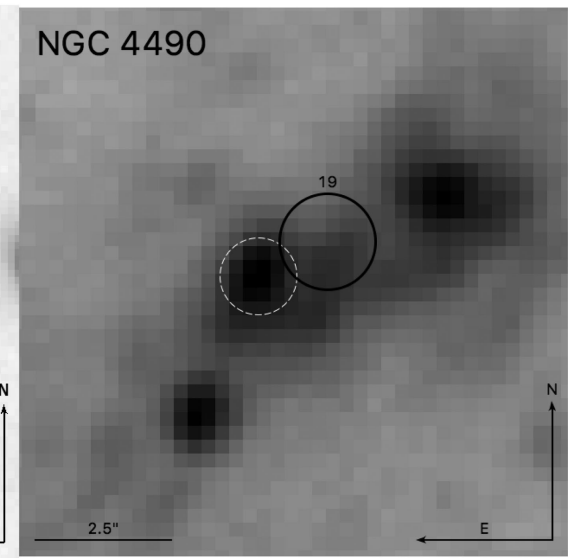

(f) NGC 4490, ULX ID 19, 3- $\sigma=0.9$ "

(d) NGC 4258, ULX ID 17, 3- $\sigma=3.4 "$

(e) NGC 4258, ULX ID 18, 3- $\sigma=1.8$ "

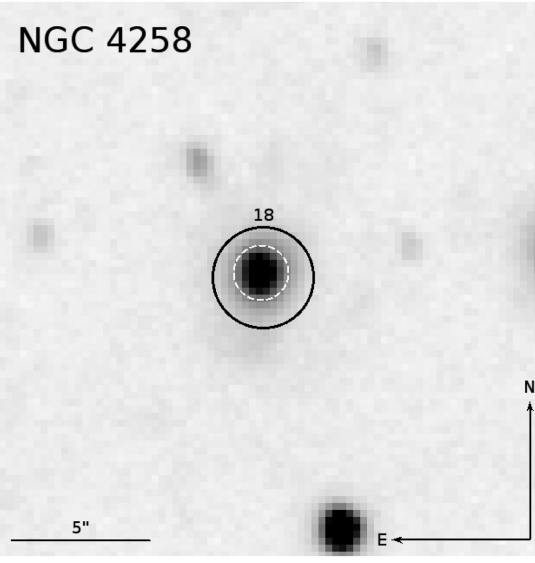

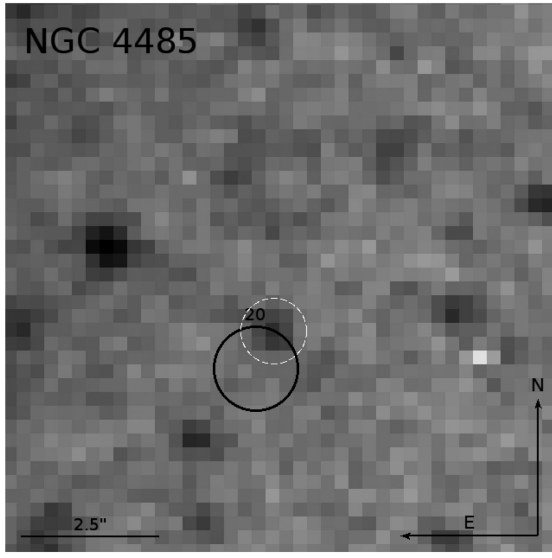

(g) NGC 4485, ULX ID 20, 3- $\sigma=0.8$ "

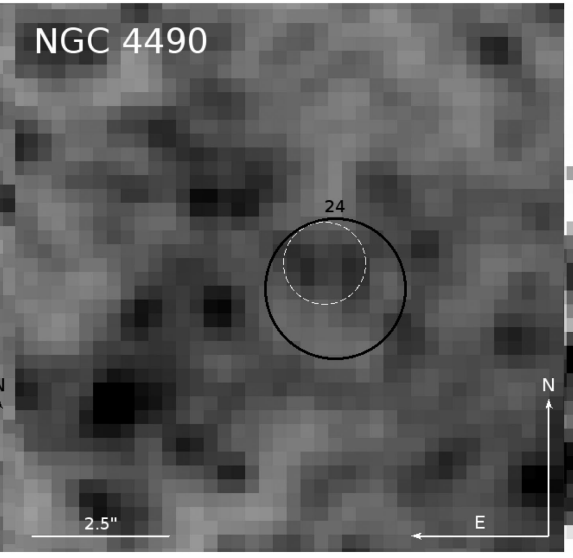

(h) NGC 4490, ULX ID 24, 3- $\sigma=1.3$ "

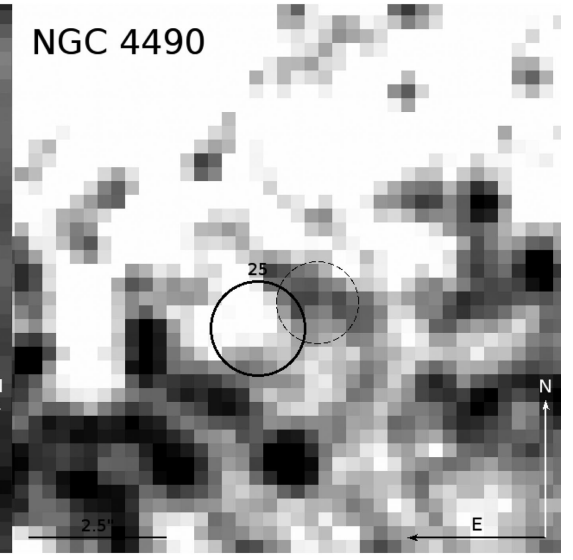

(i) NGC 4490, ULX ID 25, 3- $\sigma=0.9 "$

Figure 2. Finder charts of the ULXs with a NIR candidate counterpart. The black/white solid circles correspond to the 99.7 per cent confidence radius around the position of the ULXs, whereas the black/white dashed circles mark the candidate counterpart as detected with SEXTRACTOR. Each image provides the value for the 99.7 per cent $(3 \sigma)$ uncertainty radius. It is important to note that some faint sources seen by eye are not significantly detected by SExTRACTOR (e.g. Figs 2a, b, d, h).

This source was detected in ROSAT HRI observations by Liu \& Bregman (2005), who both interpolated the ROSAT flux into a wider $0.3-8 \mathrm{keV}$ band and corrected it for Galactic foreground column (based on a power-law spectrum with $\Gamma=1.7$, and no additional absorption) to obtain a luminosity of $1.5 \times 10^{39} \mathrm{erg} \mathrm{s}^{-1}$ for the object (based on a distance of $3.9 \mathrm{Mpc}$ ). Subsequent catalogued $X M M-$ Newton detections of this source have reported fluxes consistent with much lower luminosities, ranging between $\sim 10^{37}$ (Kong 2003) and $\sim 5 \times 10^{38} \mathrm{erg} \mathrm{s}^{-1}$ (Lin, Webb \& Barret 2012) for an improved distance of $2.73 \mathrm{Mpc}$. Lin et al. (2012) classified it as a Supersoft 


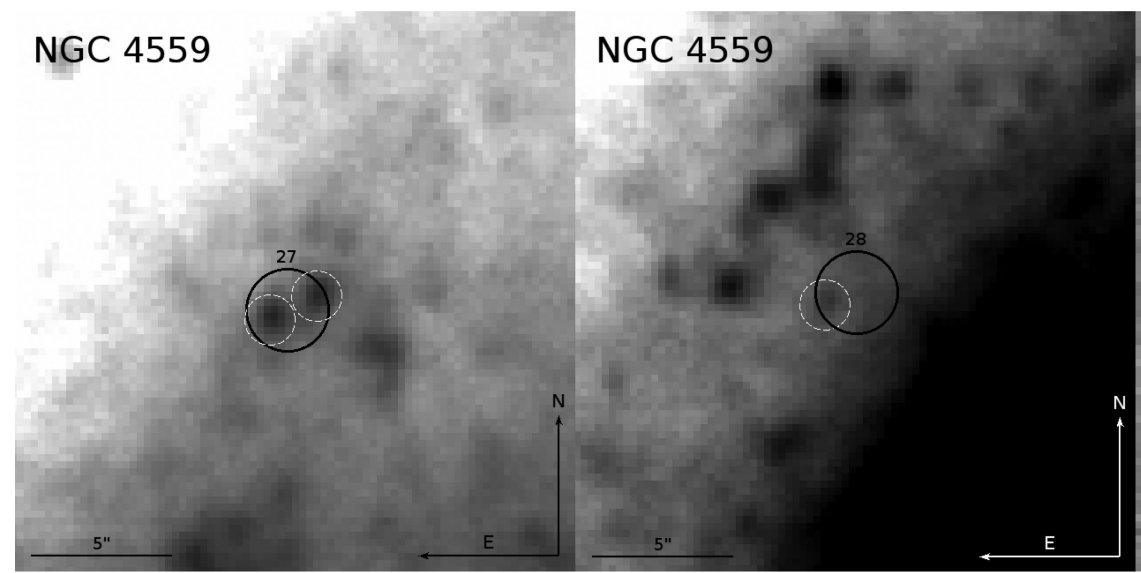

(j) NGC 4559, ULX ID 27, 3- $\sigma=1.5$ " (k) NGC 4559, ULX ID 28, 3- $\sigma=1.5$ "

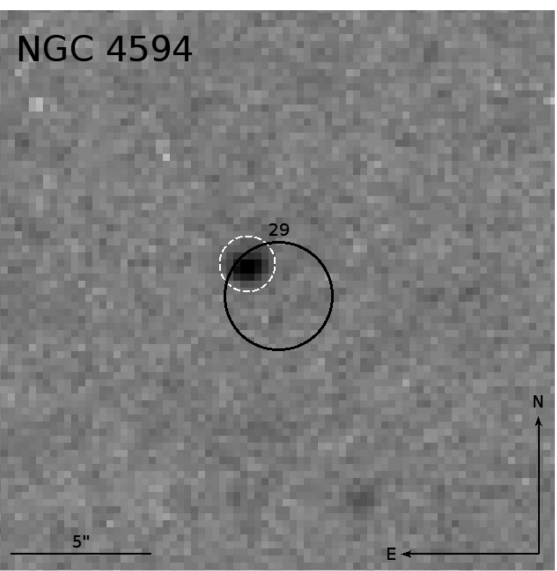

(1) NGC 4594, ULX ID 29, 3- $\sigma=1.9$ "

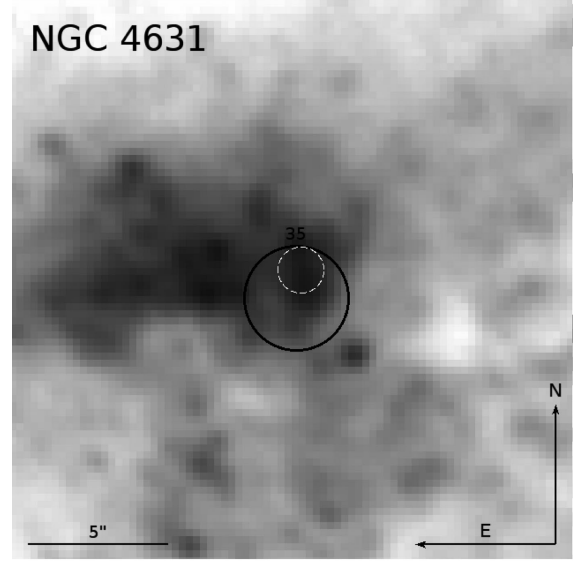

(m) NGC 4631, ULX ID 35, 3- $\sigma=1.8$ "

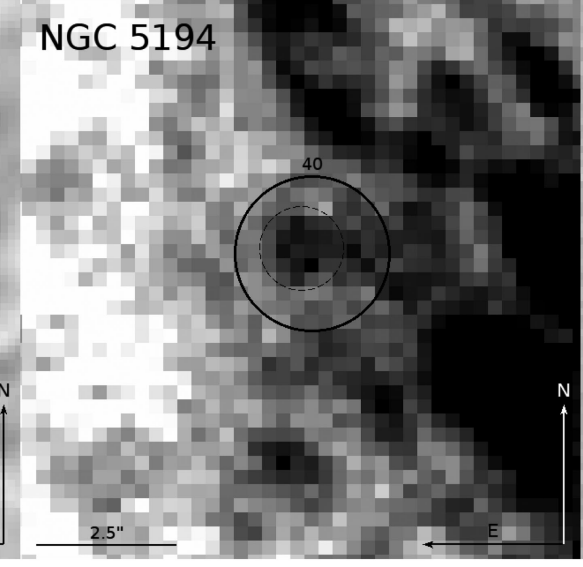

(n) NGC 5194, ULX ID 40, 3- $\sigma=1.4$ "

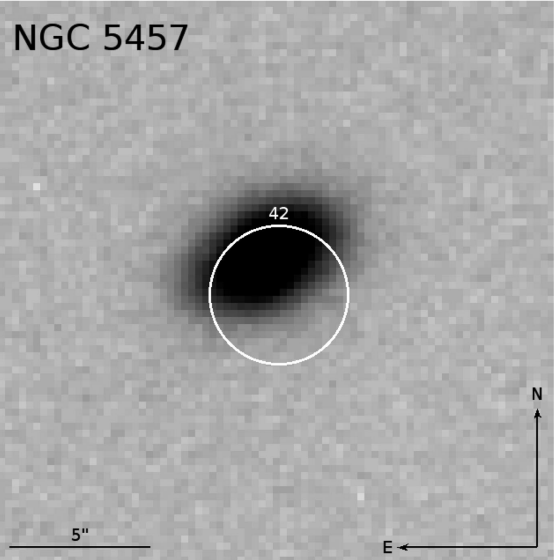

(o) NGC 5457, ULX ID 42, 3- $\sigma=2.5$ "

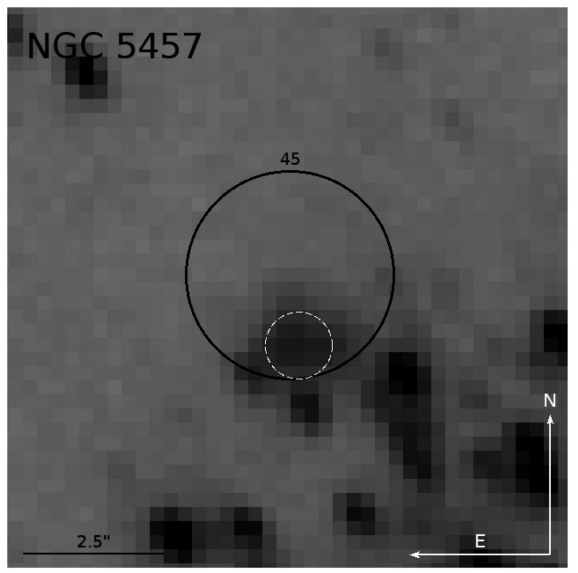

(p) NGC 5457, ULX ID 45, 3- $\sigma=1.9$ "

Figure 2 - continued.

X-ray Source, based on its hardness ratio. Additionally, Bauer, Brandt \& Lehmer (2003) determined that this X-ray source is probably not intrinsic to IC 342 , but a faint foreground star, with a position that falls just 0.3 arcsec from the bestfitting X-ray position. These newly determined lower luminosities (if in IC 342) and the possible identification as a foreground star suggest that we do not consider this source a ULX.

\subsubsection{NGC 2403 ULX ID 9}

ULX ID 9 lies in NGC 2403 (see Fig. 2b), a bulge-less galaxy. We detect a single NIR candidate counterpart of $-10.05 \pm$ $0.26 \mathrm{mag}$, consistent with an RSG absolute magnitude. Even though SExTRACTOR detected it as a single object in this crowded area, visual inspection of the image suggests that there is a second fainter NIR source. 
Table 4. NIR candidate counterparts to the ULXs listed in Table 3 and our preliminary classification as candidate RSG, AGN or stellar cluster (SC). The preliminary classification is based on their absolute magnitude, WISE colours, visual inspection of the NIR image and/or spatial extent of the candidate counterpart.

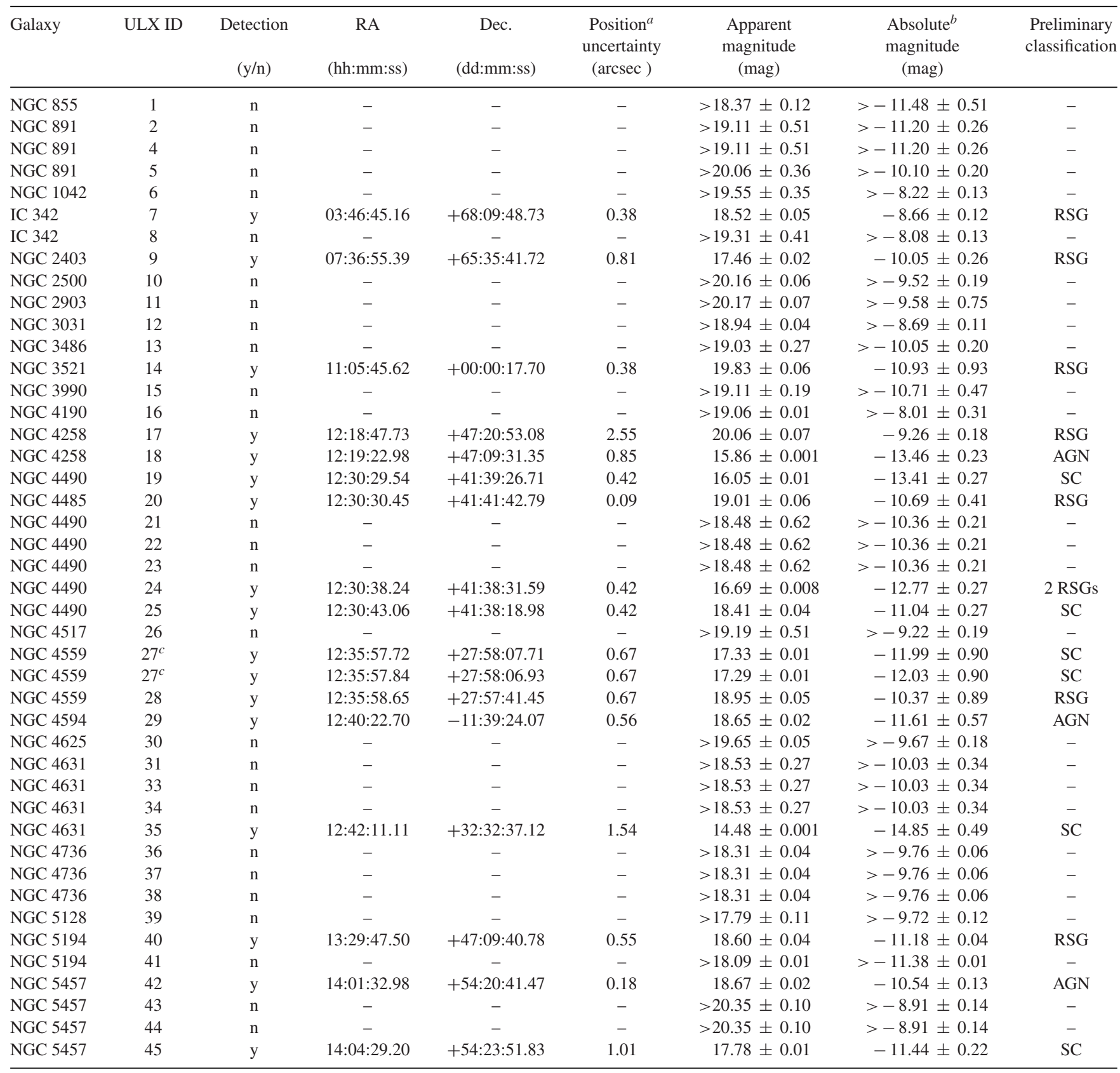

Notes. ${ }^{a} 99.7$ per cent $(3 \sigma)$ confidence radius around the position of the NIR candidate counterpart; this value is calculated taking into account the uncertainties of the astrometric correction on the NIR images and the uncertainties given by the source detection by SExTRACTOR. We added systematic uncertainties linearly and statistical uncertainties quadratically. ${ }^{b}$ Values calculated using the distance from Table 1 and the apparent magnitude, with a $1 \sigma$ uncertainty. ${ }^{c}$ Multiple NIR candidate counterparts detected.

\subsubsection{NGC 4485 ULX ID 20}

For NGC 4485 ULX ID 20, we find a $-10.69 \pm 0.41$ mag candidate counterpart (see Fig. 2g), which is consistent with an RSG. This galaxy is interacting with NGC 4490, and the galaxies have been shown to have a high star formation rate (Roberts et al. 2002).

\subsubsection{NGC 4490 ULX ID 24}

NGC 4490 ULX ID 24 is located $24 \operatorname{arcsec}(810 \mathrm{pc})$ from the galactic nucleus, and its $-12.77 \pm 0.27$ mag candidate counterpart (see Fig. 2h) seems to be an extended object, not a point source. Visual inspection suggests that this object consists of two unresolved objects, possibly two RSGs. SExTRACTOR detected it as a single object in this crowded area.

\subsubsection{NGC 4559 ULX ID 28}

We find one NIR candidate counterpart for NGC 4559 ULX ID 28 (see Fig. 2k), with an absolute magnitude of $-10.37 \pm 0.89 \mathrm{mag}$, consistent with an RSG. This ULX was observed in the optical by 
different authors. Cropper et al. (2004) did not detect any candidate counterparts for this ULX. Ptak et al. (2006) identified two candidate counterparts with $V$-band apparent magnitudes 24.4 and 23.7, and located 0.65 and 1.09 arcsec from their best-fitting X-ray positions, respectively. Given that these are smaller than our 99.7 percent confidence radius ( 1.5 arcsec), both sources would fall in our error region. However, without the coordinates of these optical candidate counterparts, we cannot be sure if our $16.69 \pm 0.008$ NIR candidate counterpart is associated with any of them.

Additionally, Vinokurov, Fabrika \& Atapin (2016) identified one optical candidate counterpart with $V$-band apparent magnitude of 24.04, located less than 0.15 arcsec from the ULX, and an estimated reddening of $\mathrm{E}(\mathrm{B}-\mathrm{V})=0.26 \pm 0.06$. Comparing their optical and our NIR source positions, we find that they are 2.314 arcsec apart, making it likely that one of the several optical sources present in the 2 arcsec box in fig. 1 of Vinokurov et al. (2016) is an RSG that we detect in the NIR. Since their error circle for the X-ray source is smaller than ours, it would exclude our RSG as a candidate counterpart.

\subsubsection{NGC 5194 ULX ID 40}

ULX ID 40 is located in NGC 5194, approximately $3 \operatorname{arcsec}(110 \mathrm{pc}$ in projection) from the star cluster [HL2008] 21287 (Hwang \& Lee 2008). We find an NIR candidate counterpart (see Fig. 2n) with absolute magnitude $-11.18 \pm 0.04 \mathrm{mag}$, consistent with an RSG absolute magnitude within the $1 \sigma$ confidence limit.

\subsection{NIR background AGN candidates}

\subsubsection{NGC 4258 ULX ID 18}

This ULX has one candidate counterpart (see Fig. 2e) within the 99.7 per cent confidence radius, with absolute magnitude of -13.46 \pm 0.23 mag, which has been observed with the Wide-Field Infrared Survey Explorer (WISE, Wright et al. 2010). Even though the WISE best angular resolution is 6.1 arcsec, the association and photometry are robust since the field of view of the ULX is not crowded. The WISE colours of this candidate are [3.4]-[4.6] $=0.78$ and [4.6]-[12] $=2.69$, making it an AGN according to the WISE colour-colour diagrams from D'Abrusco et al. (2012) and Secrest et al. (2015), which makes it likely that the X-ray source classified as a ULX is associated with the AGN.

\subsubsection{NGC 4594 ULX ID 29}

For NGC 4594 ULX ID 29 (see Fig. 21) the field of view is also not crowded, so we can rely on the WISE data to further investigate the nature of the detected candidate counterpart. This NIR source $(-11.61 \pm 0.57 \mathrm{mag})$ has colours in the WISE bands of [3.4]-[4.6] $=1.39$ and [4.6]-[12] $=2.72$, classifying it as a Seyfert galaxy according to the diagrams of D'Abrusco et al. (2012) and Secrest et al. (2015). Therefore, we deem it likely that this ULX is in reality a background AGN.

\subsubsection{NGC 5457 ULX ID 42}

We find one NIR candidate counterpart for ULX ID 42 (see Fig. 2o), with an absolute magnitude of $-10.54 \pm 0.13$ mag. Visual inspection of the NIR candidate counterpart shows an apparently extended object that is located nowhere near a star-forming region. Therefore we deem it likely that the detected source is the host galaxy of a background AGN (which would explain its absolute magnitude).

\subsection{NIR star cluster candidates}

\subsubsection{NGC 4490 ULX ID 19}

Pérez-Ramírez et al. (2011) analysed NGC 4490 ULX 19 and found one radio candidate counterpart for it, which was originally observed with Chandra (source CXOU J123029.5+413927, Roberts et al. 2002). We detect one NIR candidate counterpart inside the 99.7 per cent confidence radius around the ULX position (see Fig. 2f) with an absolute $H$-band magnitude of $-13.41 \pm 0.27$ mag. The absolute magnitude and the spatial extent of this NIR object suggest that it is a star cluster.

\subsubsection{NGC 4490 ULX ID 25}

For NGC 4490 ULX ID 25 (see Fig. 2i) we detect one NIR candidate counterpart $(-11.04 \pm 0.27 \mathrm{mag})$ which appears to be an extended source. It is possible that the NIR candidate counterpart is a star cluster, i.e. a group of unresolved RSGs. This ULX was observed before by Ptak et al. (2006), where they reported six optical candidate counterparts. However, all these optical candidate sources fall outside the 99.7 per cent confidence error radius for this ULX, and thus, none is associated with the NIR source reported here.

\subsubsection{NGC 4559 ULX ID 27}

We detect two NIR candidate counterparts for NGC 4559 ULX ID 27 (see Fig. 2j), with absolute magnitudes of $-11.99 \pm 0.90$ and $-12.03 \pm 0.90$. Even though the area is not crowded, we cannot use WISE data to investigate the nature of these two candidate counterparts as they are only 1.5 arcsec apart. Therefore, unless the colours of both candidate counterparts are such that only one source determines the WISE photometry, the WISE data will be a blend of the two sources. Visual inspection of the light distribution of the NIR image, taking into account the absolute magnitudes of the NIR sources, suggests to us that these candidate counterparts are probably star clusters.

\subsubsection{NGC 4631 ULX ID 35}

ULX ID 35 lies in a crowded area (see Fig. $2 \mathrm{~m}$ ). The NIR candidate counterpart is extremely bright $(-14.85 \pm 0.49 \mathrm{mag})$, so we rule it out as a single RSG. Since it appears to be an extended object, it could be a star cluster.

\subsubsection{NGC 5457 ULX ID 45}

We detected an NIR candidate counterpart for NGC 5457 ULX ID 45 (see Fig. 2p), with absolute magnitude of $-11.44 \pm 0.22 \mathrm{mag}$. The candidate seems to be an extended object, so it could be an unresolved young star cluster.

\section{CONCLUSIONS}

This is the second paper on our systematic search for NIR counterparts of ULXs. We observed 42 ULXs in the $H$ band and detected 
candidate counterparts for 15 of them. Of these, one has two multiple candidate counterparts. For the ULXs with non-detections, we report their limiting magnitudes.

We find that seven ULXs have NIR candidate counterparts with absolute magnitudes consistent with that of a single RSG. Two of these (NGC 3521 ULX ID 14 and NGC 4258 ULX ID 17) correspond to ULXs observed before by $\mathrm{H} 14$, and we re-observed in this work. H14 reported these ULXs as having no candidate counterparts. We find candidate counterparts because our limiting magnitudes for those two NIR images are deeper than the ones reported by H14. Another one of our seven ULXs with NIR RSG candidates correspond to NGC 4559 ULX 28, which has known optical candidate counterparts (Ptak et al. 2006; Vinokurov et al. 2016). To be completely certain of the nature of these NIR RSG candidate counterparts, spectroscopic confirmation is required.

We find eight ULXs with NIR candidate counterparts with absolute magnitudes too bright to be single stars. Indeed, some of them are extended sources. For three of these ULXs, we deem it likely that they are the host galaxies of background AGNs, based on WISE data of the field and the colour-colour diagrams of D'Abrusco et al. (2012) and Secrest et al. (2015), and their spatial extent. After visual inspection of the NIR images, we conclude that the remaining five ULXs are more likely to be star clusters.

We remove three sources from our list (see Table 3). Our more accurate positions for NGC 891 ULX ID 3 and 4, NGC 4631 ULX ID 32 and 33, show that their positions are consistent with them being the same at the $2 \sigma$ level. Thus, we conclude that the ROSAT sources are the same as the Chandra sources. The third removed source is IC 342 ULX ID 7, originally classified as a ULX by Liu \& Bregman (2005), but a new distance estimate to IC 342 renders the luminosity to fall below the ULX limit. Furthermore, it might be associated with a foreground star (Bauer et al. 2003).

We detect counterparts for 36 percent of our total sample, where 17 per cent corresponds to RSG candidates and 19 per cent to AGN/star clusters. These values are similar to the ones from H14, where 27 per cent of their ULXs present counterparts, and 18 percent are RSG candidates and 11 percent correspond to AGN/star clusters.

\section{ACKNOWLEDGEMENTS}

This research is based on observations made with the William Herschel Telescope operated on the island of La Palma by the Isaac Newton Group in the Spanish Observatorio del Roque de los Muchachos of the Instituto de Astrofísica de Canarias. We have made use of the SIMBAD data base, operated at CDS, Strasbourg, France; of the NASA/IPAC Extragalactic Database (NED), which is operated by the Jet Propulsion Laboratory, California Institute of Technology, under contract with the National Aeronautics and Space Administration; and of data obtained from the Chandra Data Archive and the Chandra Source Catalog, and software provided by the Chandra X-ray Center (CXC) in the application packages CIAO, ChIPS and Sherpa. PGJ and KML acknowledge funding from the European Research Council under ERC Consolidator Grant agreement No. 647208. TPR acknowledges funding from Science \& Technology Facilities Council (STFC) as part of the consolidated grant ST/L00075X/1.

\section{REFERENCES}

Abbott B. P. et al., 2016, Phys. Rev. Lett., 116, 061102

Bachetti M. et al., 2014, Nature, 514, 202
Bañados E. et al., 2016, Astrophys. J. Suppl. Ser., 227, 27

Bauer F. E., Brandt W. N., Lehmer B., 2003, AJ, 126, 2797

Begelman M. C., 2002, ApJ, 568, L97

Bertin E., 2006, in Gabriel C., Arviset C., Ponz D., Enrique S., eds, ASP Conf. Ser. Vol. 351, Astronomical Data Analysis Software and Systems XV. Astron. Soc. Pac., San Francisco, p. 112

Bertin E., Arnouts S., 1996, A\&AS, 117, 393

Bertin E., Mellier Y., Radovich M., Missonnier G., Didelon P., Morin B., 2002, in Bohlender D. A., Durand D., Handley T. H., eds, ASP Conf. Ser. Vol. 281, Astronomical Data Analysis Software and Systems XI. Astron. Soc. Pac., San Francisco, p. 228

Casares J., Jonker P. G., 2014, Space Sci. Rev., 183, 223

Colbert E. J. M., Miller M. C., 2006, in Novello M., Perez Bergliaffa S., Ruffini R., eds, Proc. MG10 Meeting, Observational Evidence for Intermediate-Mass Black Holes in Ultra-Luminous X-Ray Sources. World Scientific Press, Singapore, p. 530

Copperwheat C., Cropper M., Soria R., Wu K., 2005, MNRAS, 362, 79

Copperwheat C., Cropper M., Soria R., Wu K., 2007, MNRAS, 376, 1407

Cropper M., Soria R., Mushotzky R. F., Wu K., Markwardt C. B., Pakull M., 2004, MNRAS, 349, 39

D’Abrusco R., Massaro F., Ajello M., Grindlay J. E., Smith H. A., Tosti G., 2012, ApJ, 748, 68

Dobrzycki A., Ebeling H., Glotfelty K., Freeman P., Damiani F., Elvis M., Calderwood T., 1999, p. 142

Drilling J. S., Landolt A. U., 2000, in Cox A., ed., Normal Stars. SpringerVerlag, Berlin, p. 381

Ebisuzaki T. et al., 2001, ApJ, 562, L19

Elias J. H., Frogel J. A., Humphreys R. M., 1985, ApJS, 57, 91

Evans I. N. et al., 2010, ApJS, 189, 37

Fabbiano G., Zezas A., Murray S. S., 2001, ApJ, 554, 1035

Fabrika S., Ueda Y., Vinokurov A., Sholukhova O., Shidatsu M., 2015, Nat. Phys., 11, 551

Fan X. et al., 2000, AJ, 120, 1167

Feng H., Soria R., 2011, New Astron. Rev., 55, 166

Fruscione A. et al., 2006, in Silva D. R., Doxsey R. E., eds, Proc. SPIE Conf. Ser. Vol. 6270, Observatory Operations: Strategies, Processes, and Systems. SPIE, Bellingham, p. 62701V

Gao Y., Wang Q. D., Appleton P. N., Lucas R. A., 2003, ApJ, 596, L171

Gladstone J. C., Roberts T. P., Done C., 2009, MNRAS, 397, 1836

Gladstone J. C., Copperwheat C., Heinke C. O., Roberts T. P., Cartwright T. F., Levan A. J., Goad M. R., 2013, ApJS, 206, 14

Gutiérrez C. M., 2013, A\&A, 549, A81

Gutiérrez C. M., López-Corredoira M., 2006, in Meurs E. J. A., Fabbiano G., eds, IAU Symp. Vol. 230, Populations of High Energy Sources in Galaxies. Cambridge University Press, Cambridge, p. 310

Heida M. et al., 2014, MNRAS, 442, 1054 (H14)

Heida M. et al., 2015, MNRAS, 453, 3510

Heida M., Jonker P. G., Torres M. A. P., Roberts T. P., Walton D. J., Moon D.-S., Stern D., Harrison F. A., 2016, MNRAS, 459, 771

Hwang N., Lee M. G., 2008, AJ, 135, 1567

Israel G. L. et al., 2017a, Science, 355, 817

Israel G. L. et al., 2017b, MNRAS, 466, L48

Jonker P. G., Torres M. A. P., Fabian A. C., Heida M., Miniutti G., Pooley D., 2010, MNRAS, 407, 645

Jonker P. G. et al., 2012, ApJ, 758, 28

Kalogera V., Baym G., 1996, ApJ, 470, L61

King A. R., Davies M. B., Ward M. J., Fabbiano G., Elvis M., 2001, ApJ, 552, L109

Kong A. K. H., 2003, MNRAS, 346, 265

Lin D., Webb N. A., Barret D., 2012, ApJ, 756, 27

Liu J., 2011, ApJS, 192, 10

Liu Q. Z., Mirabel I. F., 2005, A\&A, 429, 1125

Liu J.-F., Bregman J. N., 2005, ApJS, 157, 59

Liu J.-F., Bregman J. N., Seitzer P., 2002, ApJ, 580, L31

Monet D. G. et al., 2003, AJ, 125, 984

Moon D.-S., Eikenberry S. S., Wasserman I. M., 2003, ApJ, 586, 1280

Motch C., Pakull M. W., Soria R., Grisé F., Pietrzyński G., 2014, Nature, 514,198 
Orosz J. A. et al., 2007, Nature, 449, 872

Patruno A., Zampieri L., 2008, MNRAS, 386, 543

Pérez-Ramírez D., Mezcua M., Leon S., Caballero-García M. D., 2011, Astron. Nachr., 332, 384

Pier J. R., Munn J. A., Hindsley R. B., Hennessy G. S., Kent S. M., Lupton R. H., Ivezić Ž., 2003, AJ, 125, 1559

Poutanen J., Fabrika S., Valeev A. F., Sholukhova O., Greiner J., 2013, MNRAS, 432, 506

Ptak A., Colbert E., van der Marel R. P., Roye E., Heckman T., Towne B., 2006, ApJS, 166, 154

Roberts T. P., Warwick R. S., Ward M. J., Murray S. S., 2002, MNRAS, 337, 677

Roberts T. P., Gladstone J. C., Goulding A. D., Swinbank A. M., Ward M. J., Goad M. R., Levan A. J., 2011, Astron. Nachr., 332, 398

Roeser S., Demleitner M., Schilbach E., 2010, AJ, 139, 2440

Schirmer M., 2013, ApJS, 209, 21

Schlegel E. M., Pannuti T. G., 2003, AJ, 125, 3025

Secrest N. J. et al., 2015, ApJ, 798, 38

Skrutskie M. F. et al., 2006, AJ, 131, 1163

Sutton A. D., Roberts T. P., Walton D. J., Gladstone J. C., Scott A. E., 2012, MNRAS, 423, 1154
Swartz D. A., Ghosh K. K., Tennant A. F., Wu K., 2004, ApJS, 154, 519

Swartz D. A., Soria R., Tennant A. F., Yukita M., 2011, ApJ, 741, 49

Tikhonov N. A., Lebedev V. S., Galazutdinova O. A., 2015, Astron. Lett., 41,239

Tully R. B., 1988, Nearby Galaxies Catalog.

Tully R. B., Rizzi L., Shaya E. J., Courtois H. M., Makarov D. I., Jacobs B. A., 2009, AJ, 138, 323

Tully R. B. et al., 2013, AJ, 146, 86

Vinokurov A., Fabrika S., Atapin K., 2016, MNRAS, preprint (arXiv: 1606.03024)

Walton D. J., Roberts T. P., Mateos S., Heard V., 2011, MNRAS, 416, 1844 Wenger M. et al., 2000, A\&AS, 143, 9

Winter L. M., Mushotzky R. F., Reynolds C. S., 2006, ApJ, 649, 730

Wright E. L. et al., 2010, AJ, 140, 1868

Wu X.-B. et al., 2015, Nature, 518, 512

Zacharias N., Finch C. T., Girard T. M., Henden A., Bartlett J. L., Monet D. G., Zacharias M. I., 2013, AJ, 145, 44

Table A1. Coordinates of the identified NIR candidate counterparts to the ULXs listed in Table 5 of H14, obtained by the 'PICK OBJECT' tool in GAIA. The classification of the NIR candidate counterparts is based on their absolute magnitudes, WISE colours, spatial extent and/or visual inspection of the NIR image. Spectra has been taken from seven sources to confirm their nature.

\begin{tabular}{|c|c|c|c|c|c|c|c|}
\hline Galaxy & $\begin{array}{l}\text { ULX name } \\
\text { in } \mathrm{H} 14\end{array}$ & $\begin{array}{c}\text { R.A } \\
\text { (hh:mm:ss) }\end{array}$ & $\begin{array}{c}\text { Dec. } \\
\text { (dd:mm:ss) }\end{array}$ & $\begin{array}{l}\text { Position }^{a} \\
\text { uncertainty } \\
(\operatorname{arcsec})\end{array}$ & $\begin{array}{l}\text { Apparent } \\
\text { magnitude } \\
(\mathrm{mag})\end{array}$ & $\begin{array}{l}\text { Absolute } \\
\text { magnitude } \\
(\mathrm{mag})\end{array}$ & Classification \\
\hline NGC 253 & J004722-252051 & $00: 47: 22.60$ & $-25: 20: 51.30$ & 0.78 & $17.2 \pm 0.03 \pm 0.5$ & $-10.5 \pm 0.03 \pm 0.5 \pm 0.10$ & $\mathrm{RSG}^{b}$ \\
\hline NGC 925 & $\mathrm{~J} 022721+333500$ & $02: 27: 21.53$ & $+33: 35: 00.70$ & 0.84 & $18.7 \pm 0.03 \pm 0.2$ & $-10.6 \pm 0.03 \pm 0.2 \pm 0.4$ & $\mathrm{RSG}^{c}$ \\
\hline NGC 925 & $\mathrm{~J} 022727+333443$ & $02: 27: 27.56$ & $+33: 34: 43.50$ & 0.84 & $20.1 \pm 0.08 \pm 0.2$ & $-9.2 \pm 0.08 \pm 0.2 \pm 0.4$ & $\mathrm{~N}^{c}$ \\
\hline NGC 1058 & $\mathrm{~J} 024323+372038$ & $02: 43: 23.28$ & $+37: 20: 42.48$ & 0.72 & $19.7 \pm 0.06 \pm 0.4$ & $-10.1 \pm 0.06 \pm 0.4 \pm 0.4$ & cRSG \\
\hline NGC 1637 & [IWL2003 68] & $04: 41: 32.9$ & $-02: 51: 26.2$ & 1.2 & $16.3 \pm 0.005 \pm 0.5$ & $-13.7 \pm 0.005 \pm 0.5 \pm 0.4$ & SC/AGN \\
\hline Holmberg II & Holmberg II X-1 & 08:19:28.94 & $+70: 42: 19.71$ & 0.66 & $19.30 \pm 0.08 \pm 0.10$ & $-8.35 \pm 0.08 \pm 0.10 \pm 0.03$ & $\mathrm{cRSG}^{e}$ \\
\hline Holmberg I & Ho I XMMI & $09: 41: 30.23$ & $+71: 12: 35.63$ & 0.42 & $17.81 \pm 0.01 \pm 0.10$ & $-10.14 \pm 0.01 \pm 0.10 \pm 0.03$ & AGN \\
\hline NGC 3627 & $\mathrm{~J} 112018+125900$ & $11: 20: 18.29$ & $+12: 59: 00.93$ & 0.72 & $20.6 \pm 1.9 \pm 0.7$ & $-9.1 \pm 1.9 \pm 0.7 \pm 0.4$ & cRSG \\
\hline NGC 4136 & $\mathrm{~J} 120922+295551$ & $12: 09: 22.63$ & $+29: 55: 50.98$ & 1.02 & $19.13 \pm 0.03 \pm 0.10$ & $-10.78 \pm 0.03 \pm 0.10 \pm 0.4$ & $\mathrm{cRSG}^{e}$ \\
\hline NGC 4136 & $\mathrm{~J} 120922+295559$ & 12:09:22.19 & $+29: 55: 59.03$ & 1.02 & $19.15 \pm 0.03 \pm 0.10$ & $-10.75 \pm 0.03 \pm 0.10 \pm 0.4$ & $\mathrm{RSG}^{c}$ \\
\hline NGC 4258 & $\mathrm{~J} 121844+471730$ & $12: 18: 43.9$ & $+47: 17: 31.0$ & 1.5 & $17.79 \pm 0.02 \pm 0.10$ & $-11.50 \pm 0.02 \pm 0.1 \pm 0.02$ & SC \\
\hline
\end{tabular}

Notes. ${ }^{a} 99.7$ per cent uncertainty radius around the position of the NIR candidate counterpart. The abbreviations are: cRSG - candidate RSG, SC - stellar cluster, N - Nebula. Confirmed nature by ${ }^{b}$ Heida et al. (2015), ${ }^{c}$ Heida et al. (2016) and ${ }^{d}$ Gutiérrez (2013). ${ }^{e}$ Spectra was taken by Heida et al. (2016), but yielded no conclusion.

This paper has been typeset from a $\mathrm{T}_{\mathrm{E}} \mathrm{X} / \mathrm{L} \mathrm{T}_{\mathrm{E}} \mathrm{X}$ file prepared by the author. 